\title{
The Moderating Effect of Urbanization on the Association between Socioeconomic Status and Physical Activity in Chinese Adults: A Cross-Sectional Study
}

\author{
Nan Chen PhD ${ }^{1}$, Chang Gyeong Kim $\mathrm{PhD}^{2}$, Kang Jae Jerry Lee PhD 3 , Jaehyun Kim PhD \\ ${ }^{1}$ Department of Global Business, Kosin University, Busan; 'Department of Chinese Studies, Pukyong National University, Busan, Korea; ${ }^{3}$ Department of Parks, Recreation \\ \& Tourism Management, North Carolina State University, Raleigh; ${ }^{4}$ Department of Recreation, Therapeutic Recreation \& Tourism, SUNY Brockport, New York, USA
}

PURPOSE: Rapid urbanization has witnessed a dramatic decrease in physical activity. The purpose of this research was to explore the vulnerable inactivity group influenced by urbanization in China.

METHODS: Cross-sectional data from the 2015 China Health and Nutrition Survey were utilized. A total of 7,319 households, including 20,914 individuals within 12 provinces, were pooled. Seven types of physical activity were included in statistical testing. Community-level urbanization was measured on an urbanicity scale consisting of 12 dimensions. Cross-level logistic regression analysis was conducted to estimate the direct and interactive effects of individual socioeconomic statuses and community-level urbanization on a leisure time physical activity (LTPA). After removing incomplete responses, 8,617 samples were selected for the statistical analyses.

RESULTS: Community-level urbanization was positively related to participation probability in the LTPA. With an increase of one standard deviation (SD) in the level of urbanization, the odds of participating in the LTPA increased by approximately six times in the most urbanized areas compared with the least urbanized areas. The effect of employment status on the probability of participation in the LTPA differed across the levels of urbanization in each community. For white-collar workers who resided in the most urbanized communities, the odds of participating in the LTPA decreased by approximately $46 \%$ compared to their peers in the least urbanized communities.

CONCLUSIONS: Community-level urbanization should be addressed when developing interventions and health policies to promote physical activity during leisure time, especially for individuals who are white-collar workers living in urbanized communities.

Key words: Community urbanization, Socioeconomic status, Leisure time physical activity, Cross-level analysis, China

\section{INTRODUCTION}

Leisure time inactivity may cause increased risks of cardiovascular disease, cancer, chronic respiratory disease, diabetes, and mental health disorders [1]. Many studies have documented that leisure time physical activity (LTPA) is an important contributor to total physical activity and can provide various health benefits [2-5]. Thus, there is a need to invest in interventions and policies that promote physical activity during leisure time. To date, rapid urbanization has witnessed some lifestyle changes in residents' health-related behaviors, including decreases in domestic activity and occupational activity [6,7]. However, regarding LTPA, some studies found that citizens who resided in more urbanized areas were more active and had higher levels of physical activity during leisure time among different geographic areas [8-10]. Nevertheless, literature examining which subpopulations have the greatest declines in LTPA with the development of urbanization is scarce. Thus, further research would yield more comprehensive findings.

Thorogood [11] indicated that health-behavior related research needs to be placed within a broader scope that emphasizes both environmental constraints and individual free choice. Given these multiple influences on LTPA, Duncan and his colleagues [12] suggested a cross-level framework that emphasizes the correlations between the microscale of people 
and the macroscale of contextual settings. Thus, LTPA needs to be examined through both macro- and micro level factors, which may enhance or constrain personal leisure participation. From this perspective, various dimensions of exogenous community urbanization, including greater availability of higher educational institutions, sanitation improvements, accessibility of amenities and sport and recreation facilities [13-16], may influence participation in LTPA. However, people with different socioeconomic statuses tend to have different social and cultural resources such as behavioral norms and knowledge, which may form distinct habits in participating in LTPA $[17,18]$. In addition, people with different socioeconomic statuses may be influenced by the context of community urbanization when partaking in LTPA. Previous research found that high levels of urbanization and income are associated with low levels of physical activity in low- and middle-income countries [19], which is different from high-income countries [20,21]. However, little is known about whether the specific domain of physical activity (e.g., LTPA) may be influenced by the interaction between urbanization and individual-level socioeconomic status (e.g., education \& employment status) at the community level. Therefore, exploring the effect of individual SES on participation in LTPA depending on community urbanization is warranted.

China, as the largest developing country in the world, has been experiencing a rapid urbanization over the past decades. The urbanization rate dramatically increased from $17.9 \%$ in 1978 to $60.6 \%$ in 2019 [22]. As China continues to urbanize, occupational physical activity continues to decrease among individuals in lower urbanicity areas [19]. Thus, participation in LTPA in lower urbanicity areas may be a critical strategy for preventing health risks in China. The purpose of this study is to examine the relationship between community-level urbanization and participation probability in LTPA using China Health and Nutrition Survey (CHNS) data in 2015. In addition, we will investigate whether the relationship between individual-level socioeconomic status and participation probability in LTPA differ with community-level urbanization. Our study contributes to identifying vulnerable inactivity groups that are influenced by the development of urbanization. The findings could provide essential information for the design of intervention strategies to promote LTPA and wellbeing in China.

\section{METHODS}

\section{Data}

This study utilized the cross-sectional data from China Health and
Nutrition Survey (CHNS) in 2015. This survey program started in 1989 by the collaboration between the Carolina Population Center at the University of North Carolina and the National Institute for Nutrition and Health at the Chinese Center for Disease Control and Prevention. The survey was conducted by a multistage, random cluster process [23,24]. In the 2015 dataset, 7,319 households, 20,914 individuals within twelve provinces were pooled. These twelve provinces contain approximately $45 \%$ of the Chinese population. After removing incomplete responses, 8,617 samples were selected for statistical analyses. Table 1 presents descriptive statistics for the outcome, predictor, and control variables.

\section{Measures}

Leisure time physical activity was measured with the question, "Do you participate in any of these activities?". Seven types of physical activities were utilized in this question including martial arts (Kung Fu, etc.); gymnastics, dancing, acrobatics; track and field (running, etc.) and swimming; walking; soccer, basketball, tennis; badminton, and volley-

Table 1. Descriptive statistics of variables

\begin{tabular}{lc}
\hline Variables & Percentage (\%) \\
\hline Leisure time physical activity & \\
Participation status (no) & $4,845(56.3)$ \\
Participation status (yes) & $3,772(43.7)$ \\
Community urbanization & \\
Quartile 4 (most urbanized) & $2,038(23.6)$ \\
Quartile 3 & $2,251(26.1)$ \\
Quartile 2 & $2,258(26.2)$ \\
Quartile1 (least urbanized) & $2,070(24)$ \\
Gender & \\
Male & $4,076(47.3)$ \\
Female & $4,541(52.7)$ \\
Marital status & \\
Never married & $468(5.4)$ \\
Married & $7,429(86.2)$ \\
Others (Divorced etc.) & $720(8.4)$ \\
Health condition & \\
Very bad & $140(1.6)$ \\
Bad & $555(6.4)$ \\
Fair & $3,403(39.5)$ \\
Good & $3,458(40.2)$ \\
Very good & $1,061(12.3)$ \\
Employment status & \\
White collar worker & $1,185(13.7)$ \\
Non-white collar worker & $2,807(32.6)$ \\
Others (retired etc.) & $4,625(53.7)$ \\
College and over & \\
Below college & $2,597(30.1)$ \\
\hline & $6,020(69.9)$ \\
\hline
\end{tabular}


ball; and other (ping pong, Tai Chi, etc.). Individual-level socioeconomic status was measured with two variables: educational attainment and employment status. Educational attainment was measured by asking respondents, "What is the highest level of education you have attained?" with responses ranging from "graduated from primary school" to "master's degree or higher" and was recoded into two categories below college and college. Employment status was measured by asking participants, "What is your primary occupation?" or "Why are you not working?". Then employment status was recoded from a number of options and grouped into three categories: employed in white collar, employed in non-white collar work, and others (seeking job, retired, homemaker, student, etc.). Age, marital status, sex, and health condition were included in the analysis as control variables. These variables were chosen because they could affect the relationship between the dependent variable and independent variables of primary interest in this study $[8,19,43]$. Age was a continuous variable, ranging from 18 years old to 75 years old.

Community-level urbanization was measured by an urbanicity scale that includes multiple contextual characteristics and has been utilized to capture the effect of urbanization on adult health behaviors from the physical, social, cultural, and economic dimensions in China $[6,7,25,26]$. This scale consisted of 12 dimensions, including community-level population density, economic activity, traditional markets, modern markets, transportation infrastructure, sanitation, communications, housing, education, diversity, health infrastructure, and social services. However, the dimension of communications was not provided in the CHNS 2015 dataset. Thus, as contributors to community-level urbanization, only $11 \mathrm{di}-$ mensions were utilized in the final statistical analysis. These indicators were standardized using the $\mathrm{z}$-score technique and were summed to provide an overall urbanization score for each community. For the 288 communities in the study areas, the median score was 2.752 (standard deviation =.419). The level of community urbanization was then converted into four categories ranging from least urbanized $<-5.134$ to most urbanized $>7.691$.

\section{Statistical Methods}

This study aimed to examine the association between cross-level predictors and LTPA in China. Cross-level logistic regression analysis was conducted to estimate the relative and interactive effects of individual socioeconomic status (education and employment status) and community-level urbanization on individual LTPA. We first calculated the ICC (intraclass correlation coefficient) to make sure that there was sufficient variation in the outcome variable at both within- and between- communities levels, which is necessary for further statistical testing [27]. The cross-level data structure considered in the present analysis consisted of 8,617 individuals (at level 1) nested within 288 communities (at level 2). According to the research purpose, the current study tested three sets of cross-level logistic models (random intercept models). These models can provide estimates of the compositional effect (individual) and the contextual effect (community) on the participation probability in LTPA. To test these hypotheses, individual socioeconomic status (individual-level fixed parameters) and community-level urbanization (community-level fixed parameters) were fitted. The intercept was assumed to be random at level 2 in all models. Generalized linear mixed modeling (GLMM) that has the extension of GLM "describes models for categorical data where the subjects are nested within groups or where repeated measures are nested within individuals", and was suggested to conduct the crosslevel logistic regression model [27]. All statistical analyses were performed using the statistical package SPSS 21.

\section{RESULTS}

Table 2 provides the fixed-effect output for the three models. The estimates for the two individual-level predictors were given in model 1 (i.e., below college, nonwhite-collar work, and others). The odds ratio suggests that the odds of participating in LTPA for adults below a college degree were reduced by approximately $22 \%$ compared with their college peers adjusted for age, sex, marital status, and health condition. Meanwhile, the odds of participating in LTPA for adults who engage in white-collar jobs and nonwhite-collar jobs were reduced by approximately $25 \%$ and $50 \%$ compared with their counterparts who are not currently employed because of work seeking, retirement, housework, and studying holding age, marital status, and health condition constant. The estimates for community-level urbanization were given in model 2 (i.e., Quartile $4=$ Most urbanized, Quartile 3, and Quartile 2). Community-level urbanization is positively related to the probability of participating in LTPA, adjusted for other predictors in the model. These results suggest that with an increase of one standard deviation (SD) in terms of urbanization, the odds of participating in LTPA increased by approximately 6times, 4times, and 2times depending on the level of urbanization compared with the least urbanized areas. For the community-level urbanization ${ }^{*}$ mployment status interaction in model 3 , the effect of employment status on the probability of participation in LTPA was different across levels of urbanization in 
Table 2. Associations between individual-level characteristics, community-level urbanization, and LTPA, results for the multi-level logistic regression models, restricted to ages 18-75, CHNS, 2015

\begin{tabular}{|c|c|c|c|}
\hline & Model 1 & Model 2 & Model 3 \\
\hline Constant & -0.13 & $-1.13^{* * *}$ & $-1.18^{* * *}$ \\
\hline \multicolumn{4}{|l|}{ Individual-level predictors } \\
\hline Education (below college $=1$ ) & $.78 a^{* *}(0.660 .91)$ & $.83^{*}(0.700 .97)$ & $.81^{* *}(0.690 .95)$ \\
\hline \multicolumn{4}{|l|}{ Employment status } \\
\hline White collar work & $.77^{* *}(0.650 .92)$ & $.75^{* * *}(0.640 .90)$ & $1.21(0.6842 .15)$ \\
\hline Non-White collar work & $.49 * * *(0.430 .57)$ & $.51^{* * *}(0.440 .58)$ & $.56^{* * *}(0.410 .76)$ \\
\hline \multicolumn{4}{|l|}{ Control variables } \\
\hline Age & $1(0.991 .00)$ & $1(0.991 .00)$ & $1(0.991 .00)$ \\
\hline Gender (female $=1$ ) & $0.99(0.921 .09)$ & $1(0.921 .09)$ & $1.01(0.921 .10)$ \\
\hline \multicolumn{4}{|l|}{ Marital status } \\
\hline Married & $1.48^{* * *}(1.191 .83)$ & $1.47^{* * * *}(1.191 .82)$ & $1.48^{* * *}(1.191 .83)$ \\
\hline Others & $1.48^{* *}(1.131 .93)$ & $1.46^{* *}(1.121 .91)$ & $1.46^{* *}(1.121 .92)$ \\
\hline \multicolumn{4}{|l|}{ Health condition } \\
\hline Bad \& very bad & $.62^{* * *}(0.490 .79)$ & $.63^{* * *}(0.490 .80)$ & $.64^{* * *}(0.500 .81)$ \\
\hline Fair & $0.84(0.691 .01)$ & $0.84(0.691 .01)$ & $0.85(0.701 .02)$ \\
\hline Good & $0.95(0.811 .12)$ & $0.96(0.811 .12)$ & $0.96(0.821 .13)$ \\
\hline \multicolumn{4}{|l|}{ Community-level urbanization } \\
\hline Quartile 4 (most urbanized) & & $6.03^{* * *}(4.478 .13)$ & $6.60^{* * *}(4.669 .34)$ \\
\hline Quartile 3 & & $4.15^{* * *}(3.075 .62)$ & $4.47^{* * *}(3.176 .29)$ \\
\hline Quartile 2 & & $1.75^{* * *}(1.262 .44)$ & $1.79^{* *}(1.242 .61)$ \\
\hline \multicolumn{4}{|l|}{ Cross-level interaction } \\
\hline Most urbanized * White collar & & & $.54^{*}(0.290 .99)$ \\
\hline Quartile 3*White collar & & & $.53^{*}(0.290 .99)$ \\
\hline Quartile $2 *$ White collar & & & $0.88(0.441 .76)$ \\
\hline \multicolumn{4}{|l|}{ Random parameters } \\
\hline Between communities & $9.59^{* * *}$ & $8.91^{* * *}$ & $8.87^{* * * *}$ \\
\hline Intraclass correlation (ICC) & $26.40 \%$ & $17.20 \%$ & $17.00 \%$ \\
\hline
\end{tabular}

A refers to Exp (coefficient); 95\% confidence interval for exp (coefficient) was presented in the parenthesis. Education (reference =college); Employment status (reference = Others), Gender (reference = male); Marital status (reference=never married); Health condition (reference=very good); Community urbanization (reference $=$ Quartile 1 least urbanized).

${ }^{*} p<0.05 ;{ }^{* *} p<0.01 ;{ }^{* * *} p<0.001$

each community. The significance of the interaction indicated that an increase in urbanization reduced the positive effect on the probability of participation in LTPA for individuals who engage in white-collar work. In other words, for white-collar workers who resided in the most urbanized communities, the odds of participating in LTPA decreased by approximately 46\% compared to their peers in the least urbanized communities, adjusted for other predictors in the model. We did not find interactive effects between community-level urbanization and nonwhitecollar work or educational attainment. The variance of a logistic distribution with a scale factor of 1.0 is $\pi^{2} / 3$, or approximately $3.29[28,29]$. Thus, for the current study, the ICC of model 1 can be calculated as 0.264 , suggesting that approximately $26.4 \%$ of the variability in participation in LTPA lies between 288 communities.

\section{DISCUSSION}

The aim of this study was to demonstrate the correlation among individual SES, community-level urbanization, and LTPA among Chinese adults using cross-level modeling. This study adds to the literature by demonstrating three major findings. First, having a lower educational level, a poor health condition, engaging in nonwhite-collar jobs, and being single may decrease the opportunity to participate in LTPA compared with their counterparts. These findings are partially consistent with previous studies that assessed the relationships between individual sociodemographic characteristics and LTPA [30-33]. Second, community-level urbanization was positively associated with LTPA after adjusting for individual-level factors. This finding lends support to prior studies that reported that the availability of comfortable sidewalks and beautiful 
scenery in urbanized areas might promote physical activity in leisure time $[8,10]$. However, some studies conducted in Europe found that urbanization level was negatively or insignificantly associated with physical active $[34,35]$. In this respect, we could conclude that the community context may be drastically different between Asian and European societies due to the fundamental differences in community formation and economic gaps in terms of regional development.

Third, community-level urbanization seemed to have decreased effects on LTPA for individuals who were white-collar workers and resided in the most urbanized areas. These findings suggest that communitylevel urbanization may influence participation probability in LTPA directly and through interactions with individual employment status. People with high socioeconomic status are assumed to have more social and cultural capital, which could stimulate their participation probability in LTPA compared with their counterparts in the Western context [36, 18]. However, this may not be the case in China. In line with the research of Attard and colleagues [19], high urbanization and income are associated with low levels of physical activity in China. This study found a negative interaction effect of high urbanization and employment status on LTPA. This result may be attributed to the following reasons. Compared with three decades ago, Chinese people enjoy relatively little leisure time currently, especially for people who reside in fast-paced and high-stress urban areas [37,38]. While urban residents have more opportunities to participate in LTPA in neighborhoods in close proximity to parks and leisure attractions, individuals with medium and high annual incomes tend to engage in passive/media and home-based activities more than their counterparts $[39,40]$. In this regard, white-collar workers who resided in urbanized areas may have many more constraints to exercise due to time limitations and heavy workloads. Previous studies have reported the vulnerability situation of rural to urban migrant workers to leisure participation $[41,42]$. However, we did not find an interactive effect between nonwhite-collar workers and community-level urbanization on LTPA. Thus, community environments that constrain individuals to engage in LTPA may be similar across all urbanization levels for nonwhitecollar workers.

We advise cautious interpretations of our findings due to some limitations in this study. The work was a cross-sectional study and was based on self-reports in the 2015 CHNS survey. The cross-sectional nature of the study has limitations in making definitive conclusions about the causality of the relationships among community-level urbanization, individual SES, and LTPA. Longitudinal data should be considered in fu- ture research to better determine the cause-effect correlation. Second, other exogenous variables could moderate the relationship between community urbanization and participation in LTPA. Third, self-reported frequency was also a limitation because of memory inaccuracy.

\section{CONCLUSION}

The purpose of this research is to explore the vulnerable inactivity group who are being influenced by the rapid urbanization in China. We found that Community-level urbanization was positively related to the participation probability in LTPA. In addition, the effect of employment status on the probability of participation in LTPA was different across levels of urbanization. For white-collar workers who resided in the most urbanized communities, the odds of participating in LTPA decreased compared to their peers in the least urbanized communities. On the basis of these findings, this study concludes that community-level urbanization should be addressed when developing interventions and health policies to promote physical activity in leisure time, especially for individuals who are white-collar workers and live in urbanized communities.

\section{CONFLICT OF INTEREST}

No penitential conflict of interest relevant to this article was reported.

\section{AUTHOR CONTRIBUTIONS}

Conceptualization: N Chen; Data curation: N Chen; Formal analysis: N Chen; Methodology: N Chen, KJ Lee; Project administration: C Kim; Writing-original draft: N Chen; Writing-review \& editing: N Chen, KJ Lee, J Kim.

\section{ORCID}

$\begin{array}{ll}\text { Nan Chen } & \text { https://orcid.org/0000-0001-8454-079X } \\ \text { Kang Jae Jerry Lee } & \text { https://orcid.org/0000-0002-5327-3235 } \\ \text { Jaehyun Kim } & \text { https://orcid.org/0000-0003-0350-1831 }\end{array}$

\section{REFERENCES}

1. Lee IM, Shiroma EJ, Lobelo F, Puska P, Blair SN, et al. Effect of physi- 
cal inactivity on major non-communicable diseases worldwide: an analysis of burden of disease and life expectancy. Lancet. 2012;380 (9838):219-29.

2. Saint-Maurice P, Coughlan D, Kelly S, Keadle S, Cook M, et al. Association of leisure-time physical activity across the adult life course with all-cause and cause-specific mortality. JAMA Netw Open. 2019;2(3): e190355. doi:10.1001/jamanetworkopen.2019.0355

3. Liu Y, Shu X, Wen W, Saito Eiko, Rahman S, et al. Association of leisure-time physical activity with total and cause-specific mortality: a pooled analysis of nearly a half million adults in the Asia Cohort Consortium. Int J Epidemiol. 2018;47(3):771-9.

4. Lee N. The benefits of exercise effect on vancer: a review. Exerc Sci. 2020;29(1):4-9.

5. Seo H. The impacts of exercise-intervention on the prevention and treatment of some types of cancer. Exerc Sci. 2021;30(1):3-7.

6. Monda K, Gordon-Larsen P, Stevens J, Popkin BM. China's transition: the effect of rapid urbanization on adult occupational physical activity. Soc Sci Med. 2007;64(4):858-70.

7. Ng SW, Howard AG, Wang HJ, Su C, Zhang B. The physical activity transition among adults in china: 1991-2011. Obes Rev. 2014;15(S1): 27-36.

8. Liu H, Walker G. The Effects of urbanization, motivation, and constraint on chinese peoplés leisure-time physical activity. Leis Sci. 2015; 37(5):458-78.

9. Reis J, Bowles H, Ainsworth B, Dubose K, Smith S, et al. Nonoccupational physical activity by degree of urbanization and U.S. geographic region. Med Sci Sports Exerc. 2004;36(12):2093-8.

10. Brownson R, Baker E, Housemann R, Brennan L, Bacak S. Environmental and policy determinants of physical activity in the United States. Am J Public Health. 2001;91:1995-2003.

11. Thorogood N. What is the relevance of sociology for health promotion? In Bunton R, Macdonald G. (Eds.), Health Promotion: Disciplines and Diversity. London: Routledge. 1992;42.

12. Duncan C, Jones K, Moon G. Health-related behaviour in context: a multilevel modelling approach. Soc. Sci. Med. 1996;42:817-30.

13. Ng SW, Norton EC, Popkin BM. Why have physical activity levels declined among Chinese adults? findings from the 1991-2006 China Health and Nutrition Surveys. Soc Sci Med. 2009;68(7):1305-14.

14. Ng SW, Popkin BM. Time use and physical activity: a shift away from movement across the globe. Obes Rev. 2012;13(8):659-80.

15. Galster GC. The mechanism(s) of neighbourhood effects: Theory, evi- dence, and policy implications. In: van Ham M, Manley D, Bailey N, Simpson L, Maclennan D. (Eds.). Neighbourhood effects research: New perspectives SE2. Netherlands: Springer. 2012;23-56.

16. Wang K, Wang X. Providing sports venues on mainland china: implications for promoting leisure-time physical activity and national fitness policies. Int. J. Environ. Res. Public Health. 2020;17(14):E5136. doi:10.3390/ijerph17145136

17. Wiltshire G, Lee J, Williams O. Understanding the reproduction of health inequalities: physical activity, social class and bourdieu's habitus. Sport Educ Soc. 2019;24(3):226-40.

18. Stuij M. Habitus and social class: a case study on socialisation into sports and exercise. Sport Educ Soc. 2015;20(6):780-98.

19. Attard SM, Howard AG, Herring AH, Zhang B, Du S, et al. Differential associations of urbanicity and income with physical activity in adults in urbanizing china: findings from the population-based china health and nutrition survey 1991-2009. Int J Behav Nutr Phys Act. 2015;12:152. doi: 10.1186/s12966-015-0321-2.

20. Bauman A, Reis R, Sallis J, Wells J, Loos R, et al. Correlates of physical activity: why are some people physically active and not others? Lancet. 2012(380):9838 DOI: 10.1016/S0140-6736(12)60735-1

21. Hallal PC, Andersen LB, Bull FC, Guthold R, Haskell W, et al. Global physical activity levels: surveillance progress, pitfalls, and prospects. Lancet. 2012;380(9838):24757. doi:10.1016/S0140-6736(12)60646-1.

22. National Bureau of Statistics of People's Republic of China. Statistical Yearbook of China 2020. 17, Mar 2021; Retrieved from http://www. stats.gov.cn/tjsj/ndsj/2020/indexch.htm

23. Popkin BM, Du S, Zhai F, Zhang B. Cohort profile: The China health and nutrition survey monitoring and understanding socio-economic and health change in China, 1989-2011. Int J Epidemiol. 2010;39(6): 1435-40.

24. Zhang B, Zhai F, Du S, Popkin, B. The China health and nutrition survey, 1989-2011. Obes Rev. 2014;15(S1):2-7.

25. Jones-Smith JC, Popkin BM. Understanding community context and adult health changes in china: development of an urbanicity scale. Soc Sci Med. 2010;71(8):1436-46.

26. Mendez MA, Popkin BM. Globalization, urbanization, and nutritional change in the developing world. The Electronic J. Dev. Agric. Econ. 2004;1(2):220-41.

27. Heck R, Thomas S, Tabata L. Multilevel modeling of categorical outcomes using IBM SPSS. New York: Routledge 2012;157,135-7.

28. Hox J. Quantitative methodology series. Multilevel analysis techniques 
and applications. Mahwah: Lawrence Erlbaum Associates Publishers 2002.

29. Hedeker D. Multilevel models for ordinal and nominal variables. In J. de Leeuw \& E. Meijers (Eds.), Hanbook of multilevel analysis. New York, NY: Springer. 2007;341-76.

30. Mäkinen T, Sippola R, Borodulin K, Rahkonen O, Kunst A, et al. Explaining educational differences in leisure-time physical activity in $\mathrm{Eu}-$ rope: the contribution of work-related factors. Scand J Med Sci Sports. 2012;22(3):439-47.

31. Chen M, Wu Y, Narimatsu H, Li X, Wang C, et al. Socioeconomic Status and physical activity in chinese adults: a report from a community-based survey in jiaxing, china. PLoS One. 2015;10(7):e0132918. doi:10.1371/journal.pone.0132918.

32. Chen N, Tsai CTL. Rural-urban divide and the social stratification in leisure participation in china: application of multiple hierarchy stratification perspective. Qual Life Res. 2020;15:1535-48. https://doi.org/10. 1007/s11482-019-09750-Z

33. Barr AL, Partap U, Young E, Agoudavi K, Balde N, et al. Sociodemographic inequities associated with participation in leisure-time physical activity in sub-saharan africa: an individual participant data metaanalysis. BMC Public Health 2020;20:927. https:/doi.org/10.1186/ S12889-020-08987-W

34. Bertrais S, Preziosi P, Mennen L, Galan P, Hercberg S, et al. Sociodemographic and geographic correlates of meeting current recommendations for physical activity in middle-aged french adults: the Supple- mentation en Vitamines et Mineraux Antioxydants (SUVIMAX) Study. Am J Public Health. 2004;94:1560-6.

35. Gast G, Frenken F, van Leest L, Wendel-Vos G, Bemelmans W. Intranational variation in trends in overweight and leisure time physical activities in the netherlands since 1980: stratification according to sex, age and urbanisation degree. Int J Obes (Lond). 2007;31:515-20.

36. Abel T. Cultural capital and social inequality in health. J Epidemiol Community Health 2008;62(7):e13. doi:10.1136/jech.2007.066159.

37. Song R, Jin Z, Li W, Wu J, He D, et al. Annual report on Chinảs leisure development (2017-2018). Beijing: Social Sciences Academic Press. 2018.

38. Qing L. A study on the distribution and utilization of workers' living time in big cities. Sociol Stud (China). 1990;1:92-102.

39. Wei X, Stodolska M. Leisure in Urban China. J. Leis. Res 2015;47(3): 373-87.

40. Liu Y, Zhang Y, Jin T, Liu Y. Spatial pattern of leisure activities among residents in beijing, china: exploring the impacts of urban environment. Sustain. Cities Soc. 2020; 52:101806.

41. Ma H. Survey on the leisure life conditions of Chinese migrant workers. In Song R. (Ed.), Annual Report on Chinảs Leisure Development. Beijing: Social Sciences Academic Press. 2011;198-216.

42. Li M, Stodolska M. Leisure as a constraint and a manifesto for empowerment: The life story of a Chinese female migrant worker. Leis Sci. 2018. DOI:10.1080/01490400.2018.1483854 